\title{
Sciences imaginaires et imaginaire de la science dans Doctor Who (2005-2014)
}

\section{François-Ronan Dubois}

\section{Q OpenEdition \\ 1 Journals}

Édition électronique

URL : http://journals.openedition.org/aes/333

DOI : 10.4000/aes.333

ISSN : 2258-093X

\section{Éditeur}

Laboratoire LISAA

\section{Référence électronique}

François-Ronan Dubois, « Sciences imaginaires et imaginaire de la science dans Doctor Who (2005-2014) ", Arts et Savoirs [En ligne], 5 | 2015, mis en ligne le 15 mars 2015, consulté le 30 avril 2019. URL : http://journals.openedition.org/aes/333 ; DOI : 10.4000/aes.333

Ce document a été généré automatiquement le 30 avril 2019.

Centre de recherche LISAA (Littératures SAvoirs et Arts) 


\title{
Sciences imaginaires et imaginaire de la science dans Doctor Who (2005-2014)
}

\author{
François-Ronan Dubois
}

1 Créée en 1963, diffusée jusqu'en 1989, reprise en 2005 et aujourd'hui toujours en production, la série télévisée britannique Doctor Who constitue un objet unique au sein du paysage télévisuel britannique et international. Il s'agit non seulement de la plus longue et de la plus populaire série de science-fiction de l'histoire de la télévision mondiale, mais également d'un cas unique où une série de genre ${ }^{1}$, et singulièrement une série de sciencefiction, rassemble une audience considérable et démographiquement variée. Ce succès a attiré l'attention des universitaires depuis près de trente ans, c'est-à-dire depuis l'étude fondatrice de Tulloch et Alvarado, en 1983, Doctor Who: The Unfolding Text ${ }^{2}$. Dans le champ pluridisciplinaire des études sur les séries télévisées, aujourd'hui largement dominé par les productions étasuniennes, Doctor Who constitue l'un des rares contrepoids à l'hégémonie d'une industrie culturelle marquée par le Nouvel Âge d'Or de la télévision américaine.

2 Ce champ d'études, qui existe donc au moins depuis les années 1980, connaît un regain d'activité depuis le début du vingt et unième siècle. Cet intérêt est sans doute motivé par ce qui est perçu comme un renouveau de la télévision de qualité aux États-Unis, notamment grâce aux séries diffusées par la chaîne câblée HBO, dont le style s'impose, par la nécessité de se démarquer dans marché hyperconcurrentiel. Si la série télévisée occupe une place sans cesse croissante dans les grilles de programmation des chaînes publiques comme privées, elle n'en demeure pas moins, en dehors des colloques de spécialistes en sociologie des média, un objet exotique. Ainsi certains chercheurs ont-ils pu observer que c'était vers le cinéma beaucoup plus que vers la série que l'on se tournait pour tenter de décrire les rapports de la culture populaire à tel ou tel thème de société . $^{2}$

3 Il est aisé pourtant de développer deux séries d'arguments, l'une relative à la réception, l'autre à la structure, qui soutiendraient l'étude des séries télévisées. D'une part, la série télévisée constitue probablement l'une des formes majeures, si ce n'est la forme majeure, 
du récit populaire contemporain ; ainsi faut-il souligner que certains épisodes de Doctor Who atteignent en Grande-Bretagne une audience remarquable de huit millions de téléspectateurs. Peu importe, de ce point de vue, la qualité intrinsèque de cet objet culturel : il suffit ici de constater que sa large diffusion fait de lui à la fois un miroir et un instrument de l'imaginaire collectif contemporain. D'autre part, la série télévisée explore de façon répétée, dans un univers sans cesse étendu, des thèmes principaux et interconnectés, de sorte que les discours qu'en produit l'interprétation travaillent avec plus de matière et d'effets que celui qui prend pour objet le médium cinématographique. En d'autres termes, un objet cinématographique est la version stable, fixée, d'un récit, contrairement à la série qui est un récit réactif, dont le développement dépend de sa réception par les téléspectateurs. Il suffit de songer qu'une saison de Doctor Who est constituée de plus d'une vingtaine d'heures de programme pour comprendre la richesse potentielle du matériau.

4 Cette variété n'est peut-être pas étrangère à une certaine versatilité du médium, qui paraît souvent pouvoir se prêter à des interprétations idéologiques parfaitement contradictoires ${ }^{4}$. Cependant, le caractère contradictoire de ces interprétations spectatoriales n'empêche pas l'élaboration de descriptions cohérentes et la production d'un sens discriminant, loin du spectre d'un parfait relativisme qui hante parfois les études culturelles ${ }^{5}$. Ainsi a-t-il été possible à Lindy Orthia de relier la représentation de la science dans les grandes époques du programme au contexte historique et idéologique dans lequel les épisodes sont inscrits ${ }^{6}$. L'objection parfois évoquée qui voudrait que la série télévisée soit une pure surface dépourvue de profondeur ou, plus exactement, une enveloppe sans contenu, et par conséquent un matériau impropre à l'exercice d'une activité interprétative ${ }^{7}$, ne tient pas face à l'analyse précise du document télévisuel, si l'on admet que la plurivocité n'est pas le signe d'une superficialité stérile.

Plutôt que de signer ce constat d'échec interprétatif, je propose donc de tenter de restituer la cohérence d'une idéologie, au sein de la nouvelle série télévisée Doctor Who. Restituer une cohérence, ce n'est pas tenter de décrypter un message que le récit télévisuel véhiculerait. S'il est vrai que certaines séries, notamment celles d'Aaron Sorkin, peuvent être didactiques, la plupart ne paraissent pas devoir démontrer telle ou telle position. Plutôt que de procéder à une démonstration, la série organise notions et concepts afin de créer, grâce à l'histoire et à l'image, des rapports entre différentes parties de l'imaginaire, afin de constituer, involontairement sans doute, un cadre pour la pensée, afin de délimiter un territoire à parcourir. C'est au traitement des sciences et des savoirs que je vais ici m'attacher.

6 La science tient une place centrale dans les histoires de Doctor Who. La science-fiction est le terrain d'accueil pour ainsi dire naturel de la science, que l'histoire de la série se déroule dans l'espace et montre des civilisations technologiquement avancées, comme c'est le cas par exemple dans Stargate SG-1 (1997-2007), Battlestar Galactica (1978-1979, 2004-2008) ou encore Farscape (1999-2005), ou bien qu'elle soit inscrite dans un présent ou un futur proche et explore les évolutions des sciences et des techniques qui nous sont familières, comme dans The X-Files (1993-2002) ou Fringe (2008-2013). Le thème scientifique n'est évidemment pas exclusif aux séries de science-fiction et peut se retrouver dans des séries médicales (House M.D., 2004-2012), policières (Bones, 2005 - en production) ou comiques (The Big Bang Theory, 2007 - en production). Il n'en demeure pas moins que les séries de science-fiction accordent une place particulière à l'intégration des avancées techno-scientifiques dans l'ensemble de la société, et développent des 
perspectives utopiques ou dystopiques, quand les séries d'autres genres tendent à localiser la science dans une partie spécifique du corps social.

7 La série Doctor Who raconte les aventures d'un alien à forme humaine, le Docteur, dernier survivant de la race des Seigneurs du Temps, qui exerçait jadis sur la galaxie une sorte de magistère moral, après avoir atteint la perfection technologique. Digne représentant de cette race éteinte, le Docteur en conserve les meilleures qualités: il est curieux et sensible, plein à la fois de compassion et de savoir. Docteur polytechnique, spécialiste en tout, toujours supérieur en expertise techno-scientifique aux ennemis qu'il rencontre, le Docteur constitue donc l'incarnation d'une certaine idée de la science. À cette version idéalisée et humaniste du scientifique s'opposent toutes les menaces techniques et scientifiques qui planent sur les personnages et, plus généralement, sur la planète Terre. Elles constituent bien souvent le nœud de l'histoire : il s'agit de sauver la planète d'une destruction certaine et de contrecarrer les effets désastreux d'une évolution technologique peu judicieuse.

8 Au personnage principal du Docteur sont constamment adjointes des compagnes de voyage, jeunes Britanniques, la plupart du temps, du Royaume-Uni contemporain. Rarement issues d'une quelconque formation universitaire, à l'exception notable de l'interne et plus tard docteur en médecine Martha Jones ${ }^{8}$, ces jeunes femmes constituent un relais du téléspectateur au sein de la diégèse: elles justifient narrativement les discours explicatifs par lesquels le Docteur présente les principaux éléments de l'intrigue et apprennent, au fil des épisodes, les règles fondamentales de l'univers qu'elles découvrent. Avec le Docteur, elles voyagent à travers le temps et l'espace. Les compagnons du Docteur sont donc généralement dépourvus de compétences techniques particulières ; quand ils en disposent, ils n'en font presque jamais la démonstration, à l'instar de Martha Jones, ils confessent la supériorité du Docteur dans le domaine, comme le fait le capitaine Jack Harkness, ou ils incarnent des rôles très secondaires dans l'ensemble de l'intrigue, comme Mickey Smith et Luke Smith.

9 Le Docteur n'en est pas pour autant le seul représentant de la science au sein de la diégèse : bien souvent, les ennemis sont également de brillants scientifiques, qu'ils soient mus par de sombres desseins ou perdent simplement le contrôle de leurs inventions ${ }^{9}$. Le ressort narratif du voyage temporel permet évidemment d'accorder une grande place au traitement de l'évolution technique et scientifique; la diversité des périodes traversées par les protagonistes de l'histoire permet de transformer celle-ci en tableaux des évolutions scientifiques et techniques. Grâce aux personnages et au contexte, la science est donc omniprésente au sein de cette série télévisée.

\section{Caractérisation des savoirs en présence}

10 Les discours savants représentés par la série sont essentiellement techno-scientifiques et inscrits dans la dynamique occidentale du progrès. Le Docteur, il est vrai, se distingue dans tous les domaines, y compris dans ceux qui relèvent des humanités (« Tooth and Claw » 2.2, «The Girl in the Fireplace» 2.4, «The Shakespeare Code » 3.2, «The Fires of Pompeii » $4.2 \&$ «The Unicorn and the Wasp » 4.7) ${ }^{10}$. Lorsqu'il rencontre Dickens, Agatha Christie ou Shakespeare, il fait la preuve de sa profonde connaissance de la culture humaine ou, plus exactement, britannique ainsi que de sa sensibilité artistique. Mais la série le montre essentiellement à l'œuvre dans le champ des sciences et des techniques, soulignant son expertise dans un grand nombre de disciplines : les télécommunications, 
l'astronomie, l'astrophysique, l'aéronautique, la robotique, la médecine, la génétique, les nanotechnologies, la chimie, la géologie, les mathématiques, etc. Cette expertise est à la fois scientifique et technique : en scientifique, il explique, assez obscurément d'ailleurs, les principes qui régissent tel phénomène naturel ou telle machine; en technicien, il intervient sur la matière, bricole, ré-agence, modifie la réalité. Avec son «tournevis sonique " (sonic screwdriver), le Docteur se présente comme un expérimentateur, non comme un froid observateur. La science du Docteur relève de la pratique plutôt que de la théorétique et le comédien qui l'incarne déploie un jeu physique, riche en gesticulations et en grimaces, dans lequel le personnage agit pendant qu'il discourt. On constate ainsi qu'en dehors de l'astrophysique peut-être, toutes les disciplines représentées dans la série connaissent des applications éminemment concrètes.

11 Les ennemis du Docteur, ponctuels ou récurrents, sont doués des mêmes compétences, à des degrés divers : les Daleks sont des experts en robotique et en génétique, tout comme les Cybermen; les Krilitanes cherchent à découvrir l'équation mathématique qui soustend l'univers, le Professeur Lazarus mène des expériences en régénération cellulaire et le Maître, ennemi récurrent du Docteur et Seigneur du Temps lui-même, est en quelque sorte son jumeau maléfique. Que l'histoire se déroule sur Terre, dans un vaisseau spatial ou sur une autre planète, elle oppose ainsi bien souvent deux groupes antagonistes qui font assaut de compétences techniques et scientifiques pour parvenir à leurs fins. Il faut à nouveau remarquer qu'à l'exclusion de Krilitanes et de leur obsession pour les mathématiques fondamentales, les ennemis du Docteur cherchent l'application pratique de leurs découvertes scientifiques. En d'autres termes, l'applicabilité d'un savoir ne constitue pas un critère d'évaluation axiologique: les gentils comme les méchants cherchent à obtenir des résultats concrets. Tout au plus peut-on observer, à ce stade de l'analyse, que la science n'est jamais présentée comme une connaissance dépourvue de conséquences.

Dans cet univers entièrement techno-scientifique, certains personnages secondaires paraissent de prime abord se soustraire à une semblable organisation de la rationalité, qu'il s'agisse d'un loup-garou («Tooth and Claw» 2.2), de sorcières ("The Shakespeare Code» 3.2), de fantômes ("The Girl in the Fireplace» 2.4), de devins («The Fires of Pompeii 4.2), de vampires ("The Vampires of Venice» 5.6), d'un trickster («The Pandorica Opens", 5.12) ou encore de Satan lui-même ("The Impossible Planet/The Satan Pit ", 2.8-9). Or, à l'exception notable de ce dernier cas, une explication cohérente est toujours donnée: ce que l'on avait pris d'abord pour les incarnations de mythes terriens sont en fait des extraterrestres aux sinistres desseins. Si fantastique il y a, pour reprendre les catégories todoroviennes ${ }^{11}$, il s'agit d'un fantastique positif, où l'incertitude est toujours levée par la rationalité, plutôt que préservée dans une inextricable indécision. L'incohérence ne dépasse guère l'épisode isolé et ne menace pas la cohérence de la saison et, à plus forte raison, de l'ensemble de la série.

13 Le champ des savoirs présentés est donc relativement homogène. De manière symptomatique, les compagnons du Docteur acquièrent peu à peu les compétences nécessaires pour affronter la menace extraterrestre et à leur étonnement premier se substitue, au fil des aventures, une expertise technique réutilisable dans les nouvelles situations qui se présentent à eux : Rose Tyler, vendeuse dans un grand magasin, finit par intégrer Torchwood, une unité spécialisée dans la lutte pour la protection de la planète («Doomsday » 2.13), Martha Jones, l'étudiante en médecine, devient membre de l'UNIT, les forces spéciales de l'ONU affectées aux affaires extraterrestres («The Sontaran 
Stratagem/The Poison Sky » 4.4-5), Donna Noble, secrétaire intérimaire dans une société de serrurerie, est pendant un instant l'égale intellectuelle du Docteur («Journeys End» 4.13) et enfin Amelia Pond se vante auprès de Néfertiti et d'un explorateur britannique du dix-neuvième siècle de pouvoir désormais manœuvrer un vaisseau spatial sans grande difficulté (« Dinosaurs on a Spaceship » 7.2). La techno-science ne constitue pas, comme sembleraient le suggérer certains épisodes limites, qu'une part de la réalité, mais bien un modèle toujours reconductible d'appréhension et de manipulation du monde.

Pour décrire ce champ, il est alors possible d'organiser la variété des sciences et des techniques évoquées en trois grands pôles: la physique, la robotique et l'ingénierie médicale. La physique est rendue nécessaire pour expliquer et crédibiliser les phénomènes relatifs aux voyages temporels. La robotique s'intéresse singulièrement au développement des intelligences artificielles susceptibles de dominer l'humanité ainsi qu'à l'hybridation humain-machine. L'ingénierie médicale, qui recoupe la robotique dans les cas de cyborg, comprend encore la génétique et différentes manipulations biologiques, par exemple destinées à s'assurer l'immortalité. Ces discours se présentent comme des développements imaginaires de sciences réellement existantes et font appel à une compréhension fondamentale, de la part du téléspectateur, des enjeux et des techniques présentés, à partir de laquelle seraient extrapolées les données du monde fictionnel. La science-fiction remplirait ici le rôle prospectif qui serait devenu le sien dans les débats publics contemporains autour de la techno-science ${ }^{12}$. C'est ce rôle qui est mis en scène dans l'épisode spécial numéro 22, un documentaire intitulé "The Science of Doctor Who ». On y voit des membres de l'équipe de production (acteurs et show runner ${ }^{13}$ ) exprimer la manière dont ils abordent la science dans la série, des extraits d'épisodes et des commentaires de scientifiques. À la fin de chaque séquence thématique (cyborgs, voyages temporels, régénération cellulaire, etc.), les scientifiques donnent une note comprise entre 1 et 5 , qui évalue la probabilité d'un tel développement technique dans le futur.

\section{Des sciences particulières à la science en général}

Ce pseudo-documentaire ne fait cependant pas illusion. Un examen attentif révèle que les interventions des scientifiques se composent toujours de trois parties: résumer le contenu du ou des épisodes auxquels ils se réfèrent, donner une présentation très succincte et très générale de la discipline scientifique en jeu et attribuer une note de plausibilité. Seule la deuxième partie constitue réellement un éclairage scientifique apporté à la compréhension de l'épisode, mais elle se contente en général d'évoquer en des termes très courants un phénomène scientifique désormais bien connu (la «théorie d'Einstein ", les nanotechnologies, les mains robotiques contrôlées par influx nerveux, etc.) et ces explications peuvent doubler le discours tenu par un personnage dans la fiction, par exemple celui de Rory Williams, qui évoque le principe fondamental des univers parallèles («The Vampires of Venice », 5.6).

En d'autres termes, les connaissances scientifiques nécessaires à la compréhension des intrigues de la série relèvent de la culture générale du téléspectateur occidental, à laquelle il peut accéder grâce à des documentaires, des émissions télévisées et des journaux d'actualité. Une compréhension précise de phénomènes complexes est inutile : le Docteur explique clairement l'effet d'une telle machine ou d'une telle expérience, sans entrer dans le détail de ses causes et de son fonctionnement. En dissimulant le processus 
intellectuel qui organise la production de ces effets, le Docteur présente la technologie comme une sorte de magie, apparemment ésotérique ; ainsi la sonde sonique multi-usage (le «tournevis sonique/sonic screwdriver ») dont il se sert pour pirater les systèmes informatiques, analyser la matière et ouvrir les portes, entre autres prouesses, fait office de baguette magique ${ }^{14}$. La présence de telle ou telle discipline dans une intrigue, la spécialisation d'un personnage dans cette discipline et la démonstration des effets d'une invention permise par cette discipline, n'impliquent en aucune manière que les principes scientifiques en soient présentés : c'est la technique qui en découle qui compte.

Il arrive certes que le Docteur se fende d'une explication pour tenter d'éclairer un phénomène particulier. Ainsi, dans l'épisode «Blink» (3.10), il se propose de permettre à Sally Sparrow une compréhension schématique des principes qui sous-tendent le voyage temporel, mais emploie finalement une formulation très vague, devenue caractéristique, pour les fans, du traitement du langage scientifique dans Doctor Who: la wibbly-wobbly timey-wimey stuff. De la même façon que se développait, dans Buffy the Vampire Slayer (1997-2003) un argot propre à la série et caractéristique de son rapport à la culture populaire ${ }^{15}$, les nouveaux Doctor Who mettent en place un discours spécifique pour traiter la techno-science, dont la principale caractéristique est d'occulter le fonctionnement interne des disciplines, des techniques et des raisonnements nécessaires à la production d'effets dans la réalité.

Pour mieux mesurer la spécificité de ce discours, on peut le mettre en rapport avec celui qui se développe dans House M. D. / Docteur House, tel qu'il est analysé par le collectif de philosophes Blitris dans La filosofia del Dr. House: Etica, logica ed epistemologica di un eroe televisivo ${ }^{16}$. La série consacre un temps considérable à explorer les différentes modalités du raisonnement médical et, comme le soulignent les auteurs, on peut y reconstruire des principes de syllogistique, des méthodes heuristiques, un parti pris épistémologique. Bref, dans son contexte réaliste, la série House M.D. offre un traitement plus approfondi du fonctionnement interne de la rationalité scientifique que ne le fait Doctor Who dans son contexte science-fictif. Dans une moindre mesure, des séries comme C.S.I. / Les Experts (2000 - en production) ou Crossing Jordan / Preuve à l'appui (2001-2007) détaillent les mécanismes des expériences scientifiques représentées avec un souci constant de réalisme, qui se caractérise par l'emprunt de séquences anatomiques visuellement caractéristiques du genre documentaire. On comprend bien, au regard de ces exemples, que l'on pourrait du reste multiplier à l'intérieur comme à l'extérieur du champ sciencefictif, le remarquable déficit de représentations proprement scientifiques dans Doctor Who ${ }^{17}$.

19 Un pareil désintérêt pour ces mécanismes internes conduit à envisager la science comme un tout indistinct dont le Docteur serait une représentation typique : il est le spécialiste polytechnique dans lequel convergent l'ensemble des disciplines scientifiques. De la même façon, les Daleks passent indifféremment des expériences génétiques (« Daleks in Manhattan/Evolution of the Daleks », 3.4-5) à la robotique (" Asylum of the Daleks » 7.1). La structure et le style de la série construisent donc une image homogène de la technoscience en tant que telle, beaucoup plus qu'ils n'explorent les différentes potentialités des disciplines spécifiques. 


\section{Un imaginaire éthique et politique de la science}

20

Il est alors tentant d'interpréter cette différence, dans l'atmosphère souvent apocalyptique de la série, comme le produit d'un discours technophobe qui ferait peser une condamnation indistincte sur l'ensemble du progrès scientifique, discours qui, du reste, ne serait pas spécifique aux nouveaux Doctor Who mais caractéristique de toute une partie de la science-fiction, comme le souligne Lindy Orthia ${ }^{18}$. Cependant, Lindy Orthia remarque avec justesse que le Docteur est tout autant un représentant de la science que les scientifiques qui tentent de détruire ou de conquérir le monde. Dès lors, les scientifiques fous ou inhumains appellent non à une condamnation de la science en général, mais la condamnation d'un certain rapport à la science. À l'inverse, le Docteur est l'incarnation du bon scientifique.

21 Le discours développé par Doctor Who n'a bien sûr rien d'original. Ce que le Docteur préconise, c'est un humanisme scientifique, c'est-à-dire une science qui ne fasse pas une apologie exclusive de la rationalité, dont la suite logique serait la condamnation des sentiments et des dispositions passionnelles. Les passions, et plus généralement l'imperfectibilité, qui est le fondement de l'humanité, donnent naissance à l'inventivité qui permet de nouvelles découvertes, tandis que la pure raison est par nature condamnée à la reproduction du même et ainsi à la stagnation (« Rise of the Cybermen / The Age of Steel » 5.6). Ce discours, développé explicitement dans les deux épisodes que je viens de citer, est encore incarné par les humains qui résistent à l'hybridation avec la machine et trouvent, dans leurs émotions, des ressources supérieures à ceux qui sont désormais leurs congénères, les cyborgs maléfiques: c'est le patriotisme dans «Army of Ghosts / Doomsday » (2.12-3), l'amour paternel dans «Closing Time» (6.12), le génie personnel dans « Asylum of the Daleks » (7.1).

À travers le refus permanent de la post-humanité, Doctor Who se fait l'avocat d'un contrôle humaniste, libéral et individualiste de la science, dans une société démocratique. Ce sont les compagnons du Docteur, dépourvus de toute connaissance scientifique, qui sont habilités à formuler des jugements éthiques sur le bien-fondé de telle ou telle technique. Ces compagnons ont toujours au moins une supériorité sur le Docteur: celle du sens moral (« The Runaway Bride », spécial 4 ; " The Angels Take Manhattan », 7.5 ; « Journeys End », 2.13). C'est au corps politique dans son ensemble, composé d'individus définis par l'humanité, à qui appartient de juger de l'opportunité de tel ou tel développement scientifique.

Bien entendu, l'antagonisme est légèrement déséquilibré. Si la série nous montre en quoi certains antagonistes sont des scientifiques, elle se contente de nous dire que le Docteur est un scientifique, sans que les procédures scientifiques et techniques ne soient déterminantes par sa caractérisation. Au contraire, le Docteur est plutôt représenté, nous l'avons vu, par son costume, dans les objets qui l'entourent, comme un magicien. Les représentations du bon scientifique au travail sont extrêmement rares, contrairement à celles qui abondent dans des séries comme Stargate SG-1, où se multiplient les races bienveillantes et technologiquement avancées. Comme prévu, décrypter un message politique et éthique s'avère tâche impossible : si la série se présente explicitement comme véhiculant un humanisme progressiste, ses représentations peuvent tout aussi bien suggérer un conservatisme pessimiste. Il ne s'agit pas de trancher, mais d'observer que le système signifiant que constitue le récit télévisuel admet les deux branches de cette 
alternative en cadrant le débat beaucoup plus étroitement qu'il n'y paraît : la science a de lourdes conséquences et il importe de les contrôler.

Il existerait donc deux grands types de techno-science imaginaire dans Doctor Who: d'un côté, une science qui serait le développement logique de celle que nous avons aujourd'hui à notre disposition et qui serait l'apanage des mauvais scientifiques, dont le public, via les compagnons, est appelé à se méfier et qu'il doit contrôler grâce au bon sens moral qu'implique nécessairement son appartenance célébrée à l'humanité; de l'autre, une science magique et ésotérique, entièrement incompréhensible pour ce même public, faite de gadgets et de poudre aux yeux, destinée à enchanter le monde et à stimuler l'inventivité de cette même espèce humaine.

En d'autres termes, c'est le rôle de la spéculation imaginaire et fictionnelle qui est double. Elle est à la fois un instrument de vigilance politique, par exemple dans les récits prospectifs qui travaillent le débat autour des nanotechnologies, et un moteur de l'inventivité scientifique, mise à l'honneur dans l'épisode documentaire « The Science of Doctor Who ». Ce discours nuancé (qui n'est pas nécessairement subtil ou inventif) est généré par la structure de la série télévisée, c'est-à-dire par la multiplication des épisodes qui permet un traitement variable d'un même thème. Charge au téléspectateur de sélectionner les éléments de cette structure pour produire une interprétation pro- ou anti-scientifique, au moment de la réception.

\section{NOTES}

1. On appelle ici «série de genre " une série déployant les mêmes univers que la littérature de genre, c'est-à-dire les séries de fantasy, de fantastique et de science-fiction, principalement.

2. John Tulloch et Manuel Alvarado, Doctor Who: The Unfolding Text, Londres, Macmillian Publishers, 1983.

3. Lincoln Geraghty, "From Balaclavas to Jumpsuits : The Multiple Histories and Identities of Doctor Who's Cybermen ", Atlantis, vol. 30, nº 1, 2008, p. 85-100.

4. Alan McKee, "Is Doctor Who Political ? ", European Journal of Cultural Studies, vol. 7, n², 2004, p. 201-217. Voir aussi Virginie Marcucci, Desperate Housewives, un plaisir coupable, Paris, Presses Universitaires de France, 2012.

5. Alan McKee, "Which is the best Doctor Who story? A case study in value judgements outside the academy", Intensities, n 1, 2001, [en ligne], (http://eprints.qut.edu.au/41991/2/41991.pdf (consulté le 31 janvier 2015).

6. Lindy A. Orthia, "Enlightenment was the choice: Doctor Who and the democratisation of science ", Thèse, Australian National University, 2010.

7. Voir par exemple Anne Cauquelin, "Séries en mouvement (remarques sur le destin de la série)", Quaderni, n 9, 1989-1990, p. 11-17. On trouvera une histoire de la perspective contraire dans Glen Creeber, "The Joy of Text? Television and Textual Analysis», Critical studies in television, vol. 1, $\mathrm{n}^{\circ} 1,2006, \mathrm{p} .81-88$.

8. À l'heure où s'écrit cet article, une nouvelle compagne, Oswin Oswald, apparait dans l'histoire. Déjà présentée dans un épisode antérieur comme un génie de la technique, son histoire personnelle est encore largement inconnue. Il faut par ailleurs noter que les premiers 
compagnons du Docteur, dans la série originelle des années 1960, sont des professeurs. Sur les compagnons du Docteur dans la première série et leur rapport à la science, voir la thèse de Lindy A. Orthia déjà citée.

9. Lindy A. Orthia, "Antirationalist critique or fifth column of scientism? Challenges from Doctor Who to the mad scientist trope ", Public understanding of science, vol. 20, n 4, 2011, p. 525-542.

10. Conventionnellement, le premier chiffre indique le numéro de la saison, le second celui de l'épisode.

11. Tzvetan Todorov, Introduction à la littérature fantastique, Paris, Le Seuil, 1970.

12. Sylvie Catellin, «Le recours à la science-fiction dans le débat public sur les nanotechnologies : anticipation et prospective ", Quaderni, $n^{\circ}$ 61, 2006, p. 13-24.

13. On appelle depuis quelques années show runner un rôle qui regroupe celui de scénariste en chef, producteur exécutif et directeur artistique. Le show runner est chargé d'assurer la cohérence de l'intrigue et de diriger les différentes parties du processus de production. Le premier show runner de la nouvelle série Doctor Who était Russell T. Davies, connu principalement pour sa série Queer as Folk (1999-2000). Le show runner actuel est Steven Moffat, qui dirige également les séries Sherlock (2010-en cours) et Jekyll (2007).

14. Michael et Margaret Rustin, “The Regeneration of Doctor Who", Children's Literature Annual n 2. The Story and the Self: Some Psychoanalytic Perspectives, sous la direction de J. Plastow, University of Herdsfordshire Press, 2008, p. 142-159.

15. Michael Adams, Slayer Slang: A Buffy the Vampire Slayer Lexicon, Oxford University Press, 2003.

16. Blitris, La filosofia del Dr. House : Etica, logica ed epistemologica di un eroe televisivo, Florence, Ponte Alle Grazie, 2007.

17. Pour une perspective plus générale, voir: Jean-Marie Chassay, «Texte et image : les signes piégés de la science, de la vulgarisation à la fiction », Image \& Narrative, n 15, 2006, [en ligne] http://www.imageandnarrative.be/inarchive/iconoclasm/chassay.htm (consulté le 29 janvier 2015).

18. Lindy Orthia, Public understanding of science, op. cit.

\section{RÉSUMÉS}

La série télévisée britannique Doctor Who est la plus longue série télévisée du patrimoine audiovisuel mondial. Au fil des décennies, depuis les années 1960, elle a accompagné l'évolution de l'imaginaire techno-scientifique de l'Ouest, à travers de la Guerre Froide, la crainte nucléaire, le développement de la génétique, les révolutions médicales et informatiques, jusqu'à nos jours. Elle a attiré à ce titre l'attention des spécialistes de la communication scientifique, qui se sont interrogés sur ses vertus vulgarisatrices et démocratiques. Loin de proposer cependant une explication constamment rationnelle des technologies et des méthodes scientifiques présentées à l'écran, Doctor Who traite la science fictive et prospective qu'elle met en scène comme une magie de conte au vocabulaire ésotérique. Devant cette série immensément populaire, qui façonne l'imaginaire commun depuis si longtemps, se pose alors la question de déterminer le discours tenu implicitement sur cette science dont le fonctionnement interne est caché ou tout simplement éludé. 
INDEX

Mots-clés : science, cinéma, Doctor Who, imaginaire

\section{AUTEUR}

\section{FRANÇOIS-RONAN DUBOIS}

Université Stendhal-Grenoble 3, Rhétorique de l'Antiquité à la Révolution \& Littératures, Idéologies, Représentations, EA 3017 \& UMR CNRS 5611, Grenoble 